\title{
Near-infrared integral-field spectra of the planet/brown dwarf companion AB Pictoris $b^{\star}$
}

\author{
M. Bonnefoy ${ }^{1}$, G. Chauvin ${ }^{1}$, P. Rojo ${ }^{2}$, F. Allard ${ }^{3}$, A.-M. Lagrange ${ }^{1}$, D. Homeier ${ }^{4}$, C. Dumas ${ }^{5}$, and J.-L. Beuzit ${ }^{1}$ \\ ${ }^{1}$ Laboratoire d'Astrophysique, Observatoire de Grenoble, 414 rue de la piscine, 38400 Saint-Martin d'Hères, France \\ e-mail: mbonnefo@obs.ujf-grenoble.fr \\ 2 Departamento de Astronomia, Universidad de Chile, Casilla 36-D, Santiago, Chile \\ 3 CRAL-ENS, 46, Allée d'Italie, 69364 Lyon Cedex 07, France \\ 4 Institut fãijr Astrophysik Göttingen, Georg-August-Universität, Friedrich-Hund-Platz 1, 37077 Göttingen, Germany \\ 5 European Southern Observatory, Casilla 19001, Santiago 19, Chile
}

Received 12 June 2000 / Accepted 22 December 2009

\section{ABSTRACT}

\begin{abstract}
Context. We have already imaged a co-moving companion at a projected separation of 2260 AU from the young star AB Pic A. Evolutionary model predictions based on $J H K$ photometry of AB Pic b suggest a mass of $\sim 13-14 M_{\text {Jup }}$, placing the object at the deuterium-burning boundary.

Aims. We aim to determine the spectral type, the surface gravity, and the effective temperature of AB Pic b. From the comparison of our absolute photometry to surface fluxes generated by atmospheric models, we also aim at deriving mass and radius estimates that are independent of evolutionary model predictions to test and refine them.

Methods. We used the adaptive-optics-fed integral field spectrograph SINFONI to obtain high-quality, medium-resolution spectra of $\mathrm{AB}$ Pic b $\left(R_{\lambda}=1500-2000\right)$ over the $1.1-2.5 \mu \mathrm{m}$ range. Our analysis relie on comparing our spectra to young standard templates and to the latest libraries of synthetic spectra developed by the Lyon group.

Results. AB Pic b is confirmed as a young early-L dwarf companion. We derive a spectral type L0-L1 and find several features indicative of an intermediate gravity atmosphere. A comparison to synthetic spectra yields $T_{\text {eff }}=2000_{-300}^{+100} \mathrm{~K}$ and $\log (g)=4 \pm 0.5$ dex. Determination of the derived atmospheric parameters of $\mathrm{AB}$ Pic b is limited by an imperfect match of current atmosphere spectra with our near-infrared observations of AB Pic $b$. The current treatment of dust settling and the missing molecular opacity lines in the atmosphere models could be responsible. By combining the observed photometry, the surface fluxes from atmosphere models and the known distance of the system, we derive new mass, luminosity, and radius estimates of AB Pic b. They independently confirm the evolutionary model predictions. We finally review the current methods used for characterizing planetary mass companions and discuss them in the perspective of future planet deep-imaging surveys that will be faced with the same limitations.
\end{abstract}

Key words. planetary systems - techniques: spectroscopic

\section{Introduction}

Understanding how planets form and evolve and which physical processes affect their atmospheric chemistry remains a major challenge in exoplanetary science, ever since the first glimpse of planetary formation revealed by the discovery of the $\beta$ Pictoris star debris disk (Smith \& Terrile 1984). Radial-velocity and transit searches have detected more than 300 exoplanets and, in some favorable cases, enabled a spectroscopic characterization of the irradiated atmospheres of transiting giant planets to be initiated (Vidal-Madjar et al. 2004; Tinetti \& Beaulieu 2009; Barnes et al. 2009). However, these techniques remain limited to the study of close-in planets with orbital radii typically smaller than 5 AU.

At wider orbits, the deep imaging technique with space telescopes (e.g. HST) or the combination of adaptive optics (AO) systems with very large ground-based telescopes (e.g. Palomar, CFHT, Keck, Gemini, Subaru, VLT) is particularly well-suited. The recent identification of young $(\leq 100 \mathrm{Myr})$, nearby $(\leq 100 \mathrm{pc})$

* Based on service-mode observations (080.C-0590(A)) collected at the European Organization for Astronomical Research in the Southern Hemisphere, Chile. stars that are members of comoving groups (Kastner et al. 1997; Zuckerman \& Song 2004; Torres et al. 2008) has offered ideal targets to look for substellar companions. Over the past years, several brown dwarf companions have been successfully detected at relatively wide ( $\geq 40$ AU) orbits from their central stars (Lowrance et al. 1999, 2000; Chauvin et al. 2003). The planetary mass companion imaged at $\sim 41$ AU from the young nearby brown dwarf 2M1207 (Chauvin et al. 2004) finally opened the way to new discoveries of other classes of targets, members of distant open clusters (Itoh et al. 2005; Neuhäuser et al. 2005; Luhman et al. 2006; Lafrenière et al. 2008; Schmidt et al. 2008), and nearby intermediate-age (0.1-1.0 Gyr) stars (Metchev et al. 2006) and to the recent breakthrough discoveries of HR 8799 bcd (Marois et al. 2008b), Fomalhaut b (Kalas et al. 2008), and the $\beta$ Pic b candidate (Lagrange et al. 2008).

In most cases, the companionship was generally confirmed by follow-up observations to show that the star and its companion share a common proper motion, whereas the companion mass was always assumed from a comparison of the companion photometry with evolutionary model predictions at the system age and distance. In some cases, high-quality spectra have allowed deviation of the companion spectral type to confirm its relatively cool atmosphere by identifying broad molecular 
Table 1. Observing log.

\begin{tabular}{|c|c|c|c|c|c|c|c|c|c|c|c|c|}
\hline UT Date & Target & Grating & $\overline{R_{\lambda}}$ & $\begin{array}{c}\text { Pre-optic } \\
\text { (mas/pixel) }\end{array}$ & $\sec z$ & $\begin{array}{c}F W H M \\
\left({ }^{\prime \prime}\right)\end{array}$ & $\begin{array}{c}\mathrm{EC}\rangle \\
(\%)\end{array}$ & $\begin{array}{l}\left\langle\tau_{0}\right\rangle \\
(\mathrm{ms})\end{array}$ & $\begin{array}{c}\text { DIT } \\
\text { (s) }\end{array}$ & NDIT & $\begin{array}{l}t_{\exp } \\
\text { (s) }\end{array}$ & Note \\
\hline $05 / 12 / 2007$ & $\overline{A B}$ Pic b & $J$ & 2000 & $25 \times 12.5$ & $1.225 / 1.221$ & $1.05 / 0.81$ & 54.4 & 60.13 & 300 & 1 & 2700 & \\
\hline $05 / 12 / 2007$ & AB Pic b & $J$ & 2000 & $25 \times 12.5$ & $1.199 / 1.198$ & $0.72 / 0.83$ & 54.6 & 71.73 & 300 & 1 & 2700 & \\
\hline $05 / 12 / 2007$ & HIP039640 & $J$ & 2000 & $25 \times 12.5$ & $1.202 / 1.200$ & $1.34 / 1.20$ & 38.9 & 120.91 & 40 & 1 & 80 & Tel STD \\
\hline $11 / 12 / 2007$ & $A B$ Pic b & $J$ & 2000 & $25 \times 12.5$ & $1.199 / 1.198$ & $0.82 / 0.81$ & 51.5 & 66.16 & 300 & 1 & 2700 & \\
\hline $11 / 12 / 2007$ & HIP023230 & $J$ & 2000 & $25 \times 12.5$ & $1.214 / 1.218$ & $0.96 / 1.24$ & 39.5 & 133.44 & 60 & 1 & 120 & Tel STD \\
\hline $12 / 11 / 2007$ & AB Pic b & $H+K$ & 1500 & $25 \times 12.5$ & 1. $200 / 1.201$ & $1.67 / 1.74$ & 14.9 & 11.15 & 300 & 1 & 2700 & \\
\hline $12 / 11 / 2007$ & HIP037963 & $H+K$ & 1500 & $25 \times 12.5$ & $1.211 / 1.210$ & $2.32 / 2.31$ & 05.4 & 6.11 & 20 & 3 & 120 & Tel STD \\
\hline
\end{tabular}

absorptions. The effective temperature $T_{\text {eff }}$ and the surface gravity $\log (g)$ can sometimes be derived using temperature- and gravity-sensitive features (Gorlova et al. 2003; Allers et al. 2007) by comparison to the template spectra of field and young dwarfs (McLean et al. 2003; Cushing et al. 2005; Lodieu et al. 2008) or using atmosphere models. However, systematic and homogeneous photometric and spectroscopic characterization of young wide planetary mass companions are essential for further constraining interior and atmosphere models that could depend on their formation mechanisms (Marley et al. 2007).

In the course of a VLT/NACO deep coronographic imaging survey of young, nearby stars (Chauvin et al. 2009), Chauvin et al. (2005a) discovered a faint commoving source at 5.5" ( 260 AU) from the young star AB Pic A (HIP30034, K2V, $V=9.16, d=45.5_{-1.7}^{+1.8} \mathrm{pc}$, Perryman et al. 1997). AB Pic A was originally identified as a member of the Tucana-Horologium association (Tuc-Hor) from its distance to the Earth, the strength of the $\lambda 6708 \mathrm{Li}$ line $(E W=260 \pm 20 \mathrm{~m} \AA$; confirmed by Mentuch et al. 2008), its filled-in $\mathrm{H} \alpha$ absorption, and the saturated $L / L_{\mathrm{X}}$ emission (Song et al. 2003, hereafter S03). S03 also obtained galactic space motions and found them to be similar to those of newly identified Tuc-Hor members. At the age of the association ( 30 Myr, see Torres et al. 2000; Stelzer \& Neuhäuser 2000; Zuckerman \& Webb 2000; Zuckerman et al. 2001; Torres et al. 2001; Scholz et al. 2007; Mentuch et al. 2008), several evolutionary models predict a companion mass of 13-14 $M_{\mathrm{Jup}}$, in agreement with the L0-L3 spectral type derived from NACO $K$-band spectroscopy. This places $\mathrm{AB} \mathrm{Pic} b$ at the planetary mass boundary ( 13.6 $\left.\mathrm{M}_{\mathrm{Jup}}{ }^{1}\right)$. Recently, Makarov (2007) has claimed that $\mathrm{AB}$ pic could be a member of the $\beta$ Pictoris association $(\beta$ Pic) because of a lower metallicity compared to Tuc-Hor members and a study of mutual conjunctions with the $\rho$ Ophiucus and Upper Sco star-forming regions. The system would be therefore considerably younger. However, this classification must be reconsidered in light of recent Viana Almeida et al. (2009) results assigning a solar metallicity to AB Pic A (Makarov, Private Com.). Alternatively, Torres et al. (2008) classified AB Pic A as a member of the 30 Myr-old Carina association from its enhanced $L / L_{X}$ emission and a convergence kinematical method described in Torres et al. (2006). In all cases, this membership revision does not modify the age of AB Pic A and $\mathrm{b}$ and the conclusion of Chauvin et al. (2005b).

We present here high-quality near-infrared spectra of the AB Pic b companion. They constitute the first results of a homogeneous survey to build an empirical library of carefully processed near-infrared spectra for young very-low mass companions. The observations of AB Pic b and the associated datareduction are presented in Sect. 2. The analysis of our spectra is presented in Sect. 3. In Sect. 4, we derive new mass, radius, and luminosity estimations using various methods to compare their

${ }_{1}$ Following the definition of the International Astronomical Union. respective limitations in the perspective of a future deep-imaging search for giant planets.

\section{Observations and data reduction procedures}

We used the SINFONI instrument (Bonnet et al. 2004), installed on the Very Large Telescope UT4 (Yepun) to conduct a spectral analysis of $\mathrm{AB}$ Pic b over the 1.1-2.45 $\mu \mathrm{m}$ range. The instrument benefits from the high angular resolution provided by a modified version of the Multi-Applications Curvature Adaptive Optic (AO) system MACAO (Bonnet et al. 2003) and of the integral field spectroscopy offered by SPIFFI (SPectrograph for Infrared Faint Field Imaging, see Eisenhauer et al. 2003). AB Pic b was observed with the $J(1.1-1.4 \mu \mathrm{m})$ and $H+K(1.45-2.45 \mu \mathrm{m})$ gratings at resolving powers of 2000 and 1500, respectively (see Table 1). The AO-loop was closed on the bright AB Pic A $(R=8.61)$. The instrument was used with the 25 mas/pixel preoptic, which limits the field of view $(\mathrm{FoV})$ to $0.8^{\prime \prime} \times 0.8^{\prime \prime}$. Small dithering of the source ( 8 different positions) increased the FoV up to $1.1^{\prime \prime}$ and allowed filtering of residual bad pixels. At the end of each observation, the telescope was nodded on the sky. Telluric standard (STD) stars were observed with identical setups without dithering right after AB Pic b. Flat fields, darks, arc-lamp, and distortion calibration frames were obtained in the days following the observations.

The whole dataset was reduced using custom $I D L$ routines and the SINFONI data reduction pipeline version 1.9.8 (Abuter et al. 2006). Routines were developed to correct raw images from negatives rows created during the bias subtraction (see The ESO data reduction cookbook; version 1.0, ESO, 2007) and to suppress an odd even effect affecting slitlet \#25. The pipeline carried out cube reconstruction from images corrected from the bias and the odd even effect. Hot and nonlinear pixels were first flagged. The distortion, the wavelength scale, and the slitlet positions were then computed on the entire detector using arc-lamp frames. Slitlet distances were measured with north-south scanning of the detector illuminated with an optical fiber. Objectsky frame pairs were subtracted, flat-fielded and corrected for bad pixels and distortions. Datacubes were finally reconstructed from clean science images and merged into a master cube. Filtering of sky line residuals resulting from sky variation was first done with the pipeline. A second iteration was performed on the slices of each individual datacube before merging then in the final master cube. Finally, an additional routine was implemented to detect and interpolate residual bad pixels.

AB Pic $b$ datacubes did not suffer from the contamination of the nearby $\mathrm{AB}$ pic A but were instead dominated by noise. Atmospheric refraction produces a shift of the source with wavelength in the FoV (from 12.5 to $\sim 70$ mas). The source was then re-centered slice per slice using polynomial-fit and subpixelic shifts with bicubic-spline magnification. The flux of the 
M. Bonnefoy et al.: Near-infrared integral-field spectra of the planet/brown dwarf companion AB Pictoris b

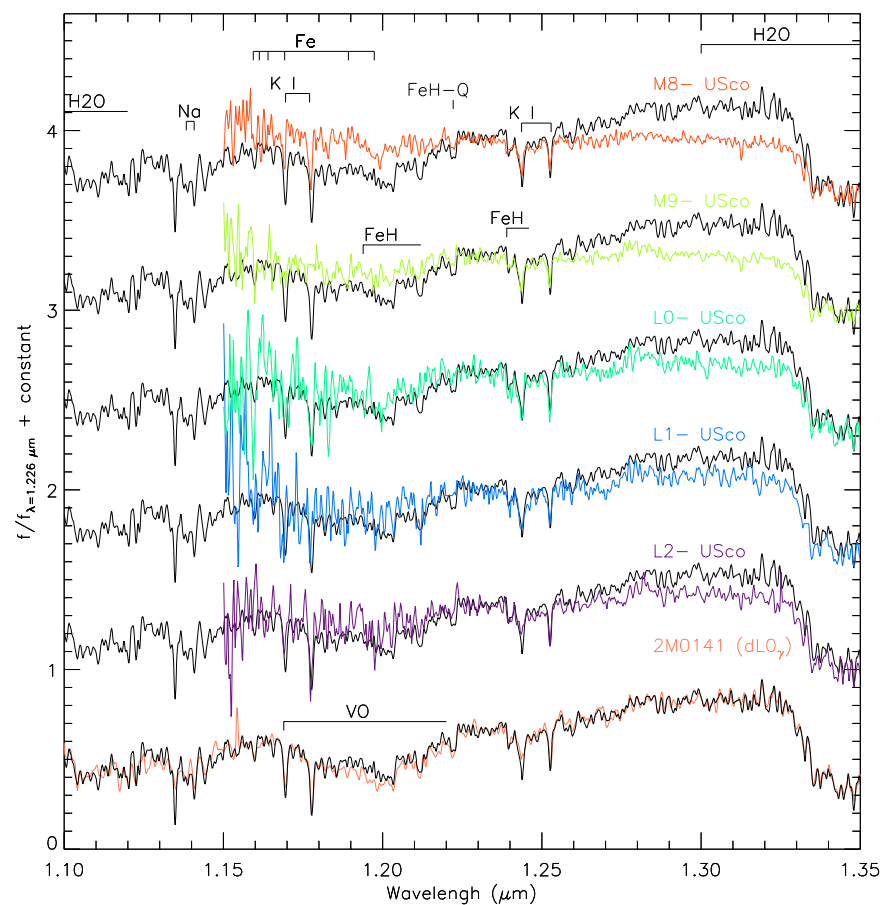

Fig. 1. $J$-band SINFONI spectrum $(1.1-1.4 \mu \mathrm{m})$ of AB Pic b (black) compared to young ( $\sim 5 \mathrm{Myr}$ ) Upper-Sco brown-dwarf spectra (color) over the $1.15-1.35 \mu \mathrm{m}$ range and to the SINFONI spectrum (salmonpink) of the young isolated object 2M0141 (classified $\mathrm{dLO} 0_{\gamma}$ ). AB Pic b and 2M0141 spectra were convolved with a Gaussian kernel to match the resolution of Upper-Sco spectra $(R=1400) \cdot \chi^{2}$ are minimized for young L1 and L2 dwarfs.

source was then integrated over an aperture minimizing the noise without introducing differential flux losses (radii of 187.5 to 512.5 mas). The STD datacubes were first divided by a black body curve at the $T_{\text {eff }}$ of the star, and the flux was collected over the entire FoV. The STD spectra were then corrected from intrinsic features using a Legendre polynomial interpolation over the surrounding continuum. AB Pic b spectra were then divided by STD spectra, normalized, and averaged to form a final spectrum.

\section{Spectral analysis}

\subsection{Empirical comparison}

The $J, H$, and $K$-band normalized spectra of AB Pic b are presented in Figs. 1-3. The signal-to-noise ratio ranges from 40 in the $H$ band to 50 in the $J$ and $K$ bands. They are compared to young M8-L2 brown dwarfs spectra of Upper Sco ( 5 Myr) (Lodieu et al. 2008, hereafter L08) at identical resolutions. In addition, $H$ and $K$-band spectra are compared to the spectrum of the $\mathrm{L}_{-2}^{+1}$ companion candidate to 1RXS J160929.1-210524, member of Upper Sco (Lafrenière et al. 2008). In agreement with our expectations, the AB Pic b spectra are typical of young late$\mathrm{M} /$ early-L dwarfs. The triangular shape of the $H$-band (Lucas et al. 2001; Luhman et al. 2004), the bumpy $K$-band, the reduced strength of alkali lines ( $\mathrm{Na}$ I at $1.138 \mu \mathrm{m}, \mathrm{K}$ I doublets at $1.169 / 1.177$ and $1.243 / 1.253 \mu \mathrm{m}$ and the $\mathrm{K} \mathrm{I}$ line at $1.517 \mu \mathrm{m})$, and of $\mathrm{FeH}$ absorptions over the $J$ and $H$ bands (Wallace \& Hinkle 2001; Cushing et al. 2003) all results from the intermediate surface gravity of the companion. This is lillustrated in Fig. 4, where the $J$-band spectrum of $\mathrm{AB}$ Pic b shows intermediategravity KI doublets compared to the low and high-gravity spectra of the IO Vir giant star and field dwarfs.

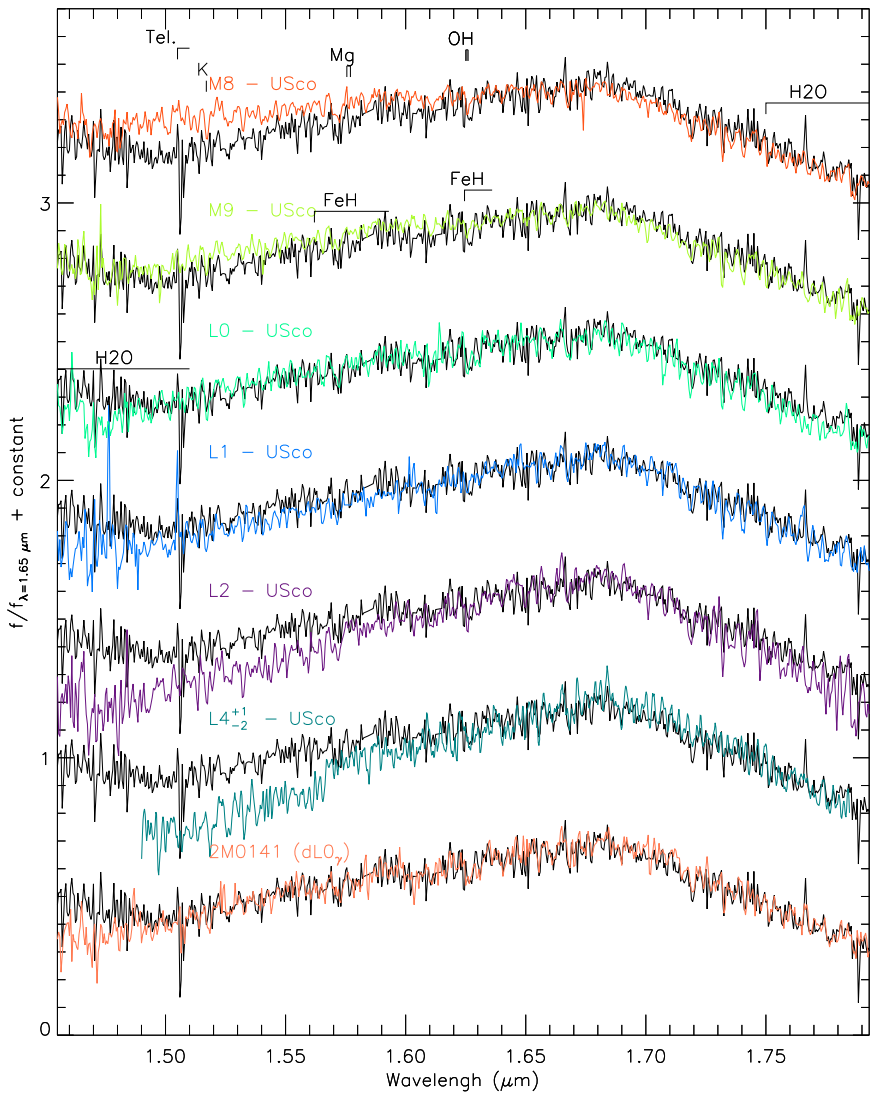

Fig. 2. $H$-band SINFONI spectrum (1.45-1.8 $\mu \mathrm{m})$ of AB Pic b (black) compared to young ( $\sim 5 \mathrm{Myr})$ Upper-Sco brown-dwarf spectra (color) over the $1.5-1.8 \mu \mathrm{m}$ range and to the SINFONI spectrum (salmonpink) of the young isolated object $2 \mathrm{M} 0141$ (classified $\mathrm{dL} 0_{\gamma}$ ). $\chi^{2}$ are minimized for a young L0 dwarf. The triangular profile of our spectrum results from reduced collision-induced absorption of $\mathrm{H}_{2}$ observed in young objects.

Table 2. Spectral indexes and associated spectral types for AB Pic b.

\begin{tabular}{ccccccc}
\hline \hline Index & $\mathrm{H}_{2} \mathrm{OA}$ & $\mathrm{H}_{2} \mathrm{O}-1$ & $\mathrm{H}_{2} \mathrm{O}-2$ & $\mathrm{H}_{2} \mathrm{O}$ & $\mathrm{K} 1$ & $\mathrm{~K} 2$ \\
\hline Value & 0.57 & 0.61 & 0.89 & 1.14 & 0.1 & 0.04 \\
Sp. type & $\mathrm{L} 2 \pm 2$ & $\mathrm{~L} 2 \pm 2$ & $\mathrm{~L} 0 \pm 2$ & $\mathrm{M} 9 \pm 2$ & $L_{\mathrm{pec}}$ & $L_{\mathrm{pec}}$ \\
\hline
\end{tabular}

To assign a spectral type, we used two different approaches. We first compared our spectra to those of young M8-L4 brown dwarfs (from L08 and Lafrenière et al. 2008) classified in the near-infrared. A $\chi^{2}$ minimization was obtained for young L1 and L2 dwarfs in the $J$ band, L0 dwarf in the $H$ band and, L0 and L2 dwarfs in the $K$ band. L2 and L4 were excluded from the visual comparison of the pseudo-continuum shape in the H-band. We also used spectral indexes to measure the depth of water absorptions at $1.34 \mu \mathrm{m}\left(\mathrm{H}_{2} \mathrm{OA}\right.$ and $\mathrm{H}_{2} \mathrm{O}-1$; see McLean et al. 2003; Slesnick et al. 2004), $1.5 \mu \mathrm{m}\left(\mathrm{H}_{2} \mathrm{O}\right.$, see Allers et al. 2007), and $2.04 \mu \mathrm{m}\left(\mathrm{H}_{2} \mathrm{O}-2\right.$, see Slesnick et al. 2004). These absorbtions are only slightly sensitive to the age. These indexes confirmed that AB Pic b has a near-infrared spectral type between a LO and L1 (Table 2). The K1 and K2 indexes measuring the strength of the $\mathrm{H}_{2} \mathrm{O}$ band from 2.0 to $2.14 \mu \mathrm{m}$, and of the $\mathrm{H}_{2}$ absorption around $2.2 \mu \mathrm{m}$ respectively (Tokunaga \& Kobayashi 1999, hereafter T99) finally discriminated AB Pic b $\left(L_{\mathrm{pec}}\right)$ from old field dwarfs (see Fig. 4 of T99; computed for young BD and field dwarfs). 


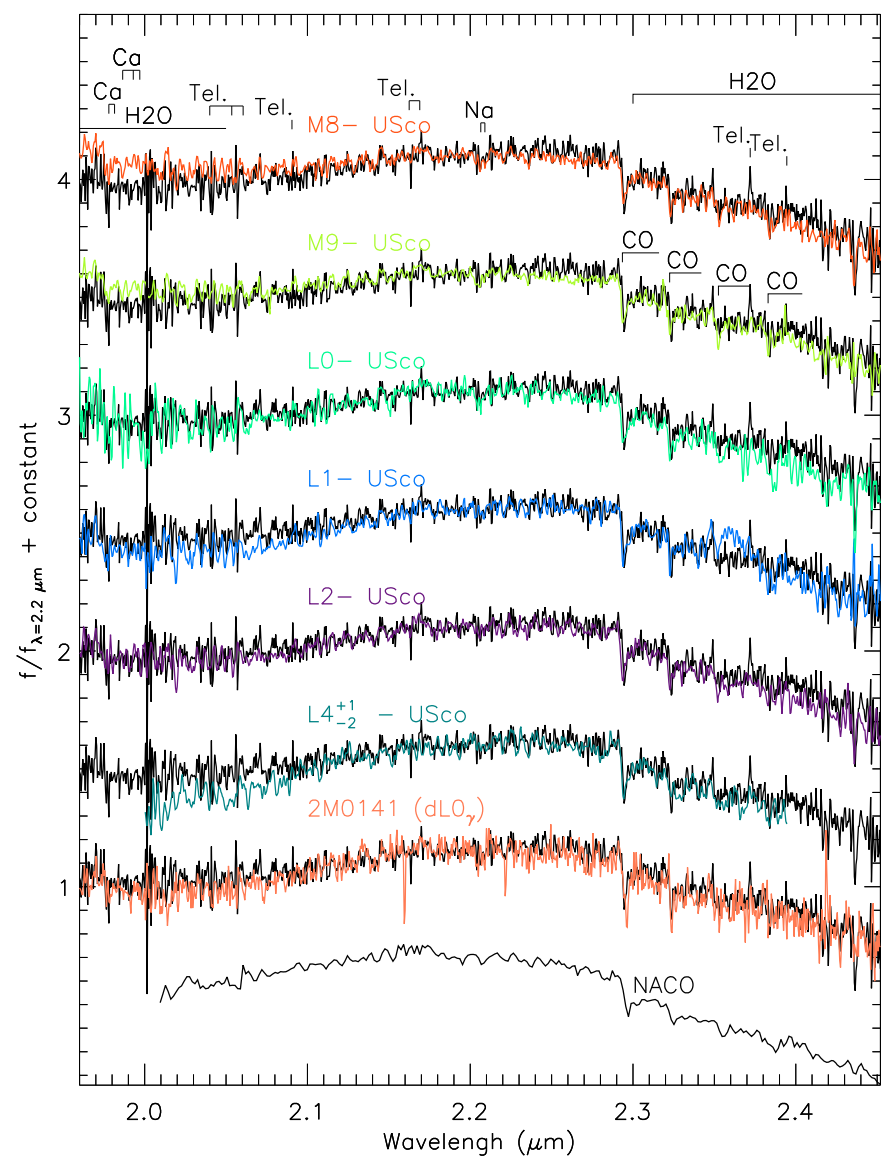

Fig. 3. $K$-band SINFONI spectrum of AB Pic b (black) compared to young ( $\sim 5 \mathrm{Myr}$ ) Upper-Sco brown-dwarf spectra (color) and to the young isolated object $2 \mathrm{M} 0141$ (classified $\mathrm{dL}_{\gamma}$ ). $\chi^{2}$ are minimized for young L0 and L2 dwarfs. The shape is not reproduced well by old field dwarfs. The low-resolution $\left(R_{\lambda}=550\right)$ NACO spectrum is overplotted at the bottom for comparison.

However, near-infrared spectral types are not necessarily consistent with those infered at optical wavelenghs where a homogeneous classification scheme for field and young brown dwarfs exists. We noticed that the normalized spectra of the L08 sample tend to be bluer than those of AB Pic b in the $J$ band. AB Pic b normalized $H+K$ band spectrum also appear overluminous in the $K$ band (or underluminous in the $H$-band). The only exceptions are UScoJ163919-253409 and UScoJ160918222923 (classified as L1 by L08) for which a good simultaneous fit in the $J$ and $H+K$ bands is achieved. This discrepancy could be related to peculiar dust properties, differences in dust opacities, binarity, or even extinction by a disk (Luhman et al. 2007). However, Herczeg et al. (2009) reveals that the classification of L dwarfs given in L08 could be strongly revised using optical spectra. We then ultimately decided to compare our spectra to those of the young L0 dwarf 2MASS J014158234633574 (Kirkpatrick et al. 2006, hereafter K06). This dwarf (hereafter 2M0141) has been classified in the optical following the scheme of Kirkpatrick (2005). The excellent match of our spectrum with that of $2 \mathrm{M} 0141$ in the $J$, and $H+K$ bands (see Figs. 1-3) leads us to assign a final spectral type L0-L1 to AB Pic b.

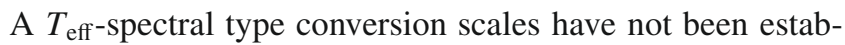
lished for young early-L dwarfs yet. However, the Luhman et al. (2003) scale valid for young M dwarfs ( 2 Myr) gives an upper limit fot the effective temperature of $2400 \mathrm{~K}$. L08 give in

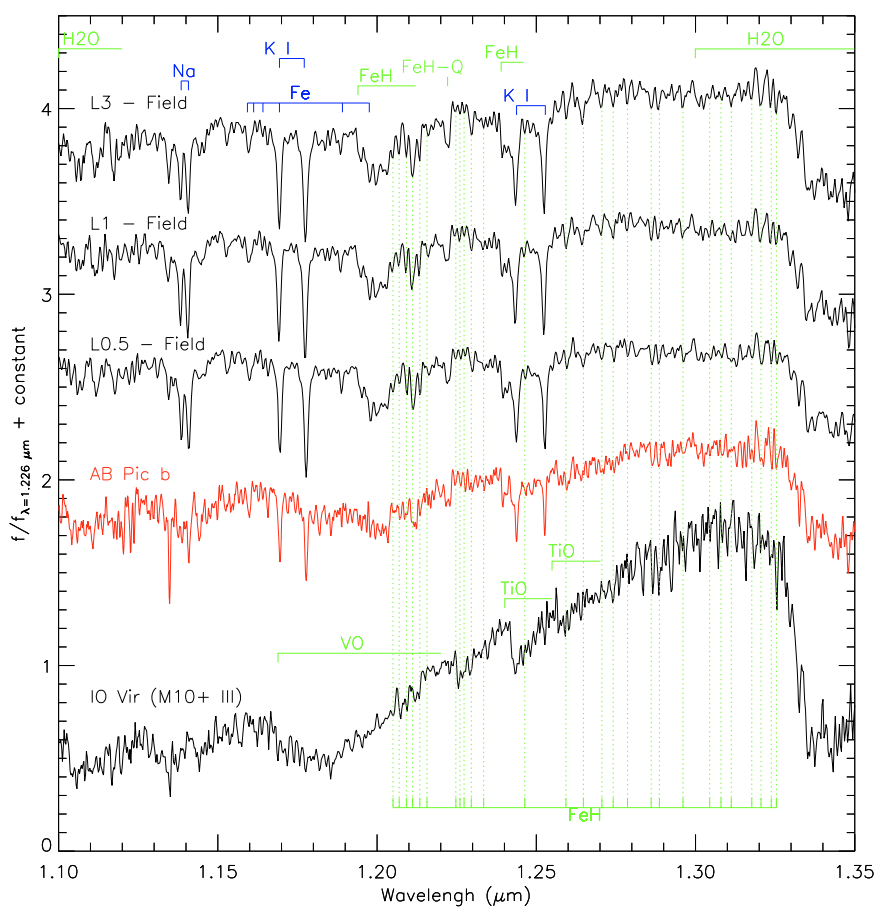

Fig. 4. $J$-band SINFONI spectrum of AB Pic b (red) compared to the spectrum of a late-type $M$ giant (IO Vir) and to field dwarf spectra (Cushing et al. 2005) over the 1.1-1.35 $\mu \mathrm{m}$ range. Atomic features are plotted in blue and molecular absorptions in green.

addition an estimation of $2000 \mathrm{~K} \lesssim T_{\text {eff }} \lesssim 2300 \mathrm{~K}$ for L0 to L1 young brown-dwarfs of the Upper Sco association.

\subsection{Comparison to atmosphere grids}

We used the AMES-Dusty00 library of synthetic spectra ${ }^{2}$ (Allard et al. 2001) that incorporates formation of dust in the atmosphere for $T_{\text {eff }} \lesssim 2600 \mathrm{~K}$. Spectra were convolved by a Gaussian kernel to match the resolution of SINFONI, interpolated on the $\mathrm{AB}$ Pic $\mathrm{b}$ wavelength grid, and normalized at $1.226 \mu \mathrm{m}, 1.56 \mu \mathrm{m}$, and $2.2 \mu \mathrm{m}$ in the $J, H$, and $K$ band. The $\mathrm{H}_{2} \mathrm{O}$ absorptions from 1.32 to $1.60 \mu \mathrm{m}$ and from 1.75 to $2.20 \mu \mathrm{m}$ are known to be overestimated in the models (Leggett et al. 2001; Lucas et al. 2001; Kirkpatrick et al. 2006). We then compared the AB Pic b spectral continuum to spectral models over the 1.1-1.34 $\mu \mathrm{m}$ zone using a classical, weighted least-square method. The analysis led to $T_{\text {eff }}=2000 \pm 100 \mathrm{~K}$ and $\log (g)=$ $4.0 \pm 0.5 \mathrm{dex}$. The fit was still visually acceptable for $T_{\text {eff }}$ down to $1700 \mathrm{~K}$ and up to $2100 \mathrm{~K}$ (see Figs. 5 and 6). Comparatively, K06 find similar atmospheric parameters for 2M0141.

The $K$-band was not properly fitted at $T_{\text {eff }}=2000 \mathrm{~K}$, and higher effective temperatures $\left(T_{\mathrm{eff}}=2500 \mathrm{~K}\right)$ could match the observed depth of $\mathrm{CO}$ overtones at $\lambda \geqslant 2.3 \mu \mathrm{m}$ better and the shape of the pseudo-continuum. The case of $2 \mathrm{M} 0141$ is likely to be similar (see the lower panel of Fig. 9 of K06). Finally, the $K$-band of AB Pic b was reproduced better at $T_{\text {eff }}=2000 \mathrm{~K}$ and $\log (g)=4.0$ dex using the most recent SETTL08 library. This library incorporates an updated bank of molecular opacities (BT), a more realistic mixing length parameter $(\alpha=2)$, and settling and replenishment of the dust in the photosphere.

Finally, the $H$-band is marginally reproduced by AMESDusty00 and SETTL08 models. These non-reproducibilities are

\footnotetext{
2 The spectra can be generated on demand on http://phoenix. ens-lyon. fr/simulator/index . faces
} 
M. Bonnefoy et al.: Near-infrared integral-field spectra of the planet/brown dwarf companion AB Pictoris b

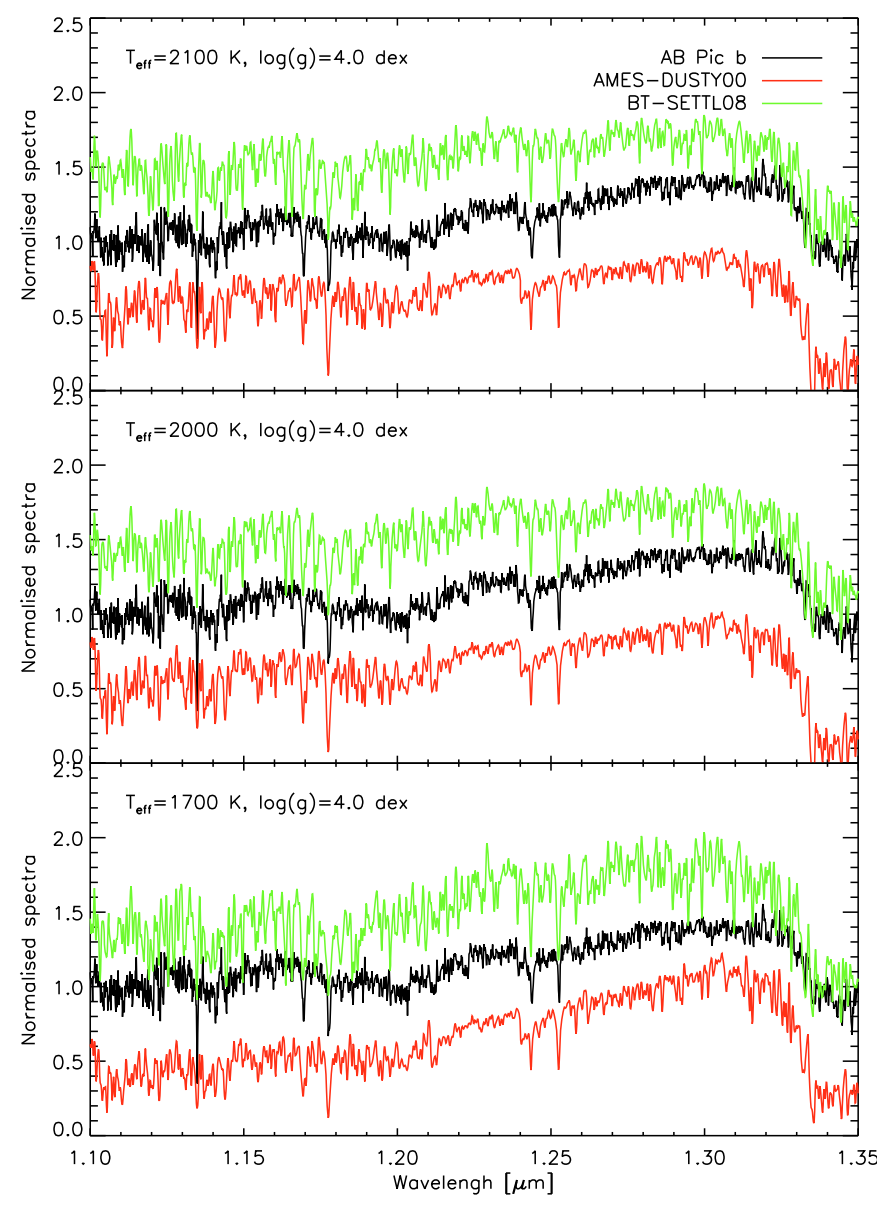

Fig. 5. Comparison of the AB Pic b $J$ band spectrum to the synthetic spectra of the AMES-Dusty00 and SETTL08 libraries at $\log (g)=$ $4.0 \mathrm{dex},[\mathrm{M} / \mathrm{H}]=0 \mathrm{dex}$, and $T_{\text {eff }}=1700 \mathrm{~K}, 2000 \mathrm{~K}$ and $2100 \mathrm{~K}$.

strengthened in SETTL08 spectra by an incorrect representation of the thermal structure of the photosphere. This effect is also responsible for the overestimation of several narrow absorptions in the $J$ band (see Fig. 5).

Nordström et al. (2004) reports AB Pic A as a metal poorstar with $\mathrm{Fe} / \mathrm{H}=-0.64 \pm 0.12$ dex. However, this estimation relies on a relation based on color indexes that could be biased for very young objects. More recently, Viana Almeida et al. (2009, hereafter V09) have measured spectroscopically a $\mathrm{Fe} / \mathrm{H}=+0.07$ dex. At a distance of $\sim 260 \mathrm{AU}$, one probable formation mechanism for $\mathrm{AB} \mathrm{Pic} b$ is via a binary-like process, i.e. with initial abundances similar to those of $\mathrm{AB}$ pic $\mathrm{A}$. We then compared our spectrum to SETTL08 models with $\mathrm{Fe} / \mathrm{H}$ between -0.5 and 0.0 and $T_{\text {eff }}=2000_{-300}^{+100} \mathrm{~K}$ to test their influence on our fits. The models indicate that subsolar abundances do not change the $J$-band pseudo-continuum but increase the depth of the $\mathrm{K} \mathrm{I}$ doublets at $1.169 / 1.177 \mu \mathrm{m}$ and $1.243 / 1.253 \mu \mathrm{m}$ (see Fig. 7). The $K$-band shows weaker $\mathrm{CO}$ overtones and reduced $\mathrm{H}_{2} \mathrm{O}$ absorptions (see Fig. 8). This effect does not affect our $T_{\text {eff }}$ estimation but could lead to slightly $\operatorname{lower} \log (g)$ estimates. A more extensive analysis of our spectra with dedicated synthetic spectra libraries could add new constraints on individual abundances of AB Pic b.

In conclusion, both AMES-Dusty00 and SETTL08 libraries yield $T_{\text {eff }}=2000_{-300}^{+100} \mathrm{~K}$ and $\log (g)=4.0 \pm 0.5$ dex for AB Pic b, for solar and subsolar $(\mathrm{Fe} / \mathrm{H}=-0.5)$ metallicities. In comparison, Mohanty et al. (2007) (hereafter M07) fit near-infrared colors and absolute photometry of AB Pic b with SETTL05 and

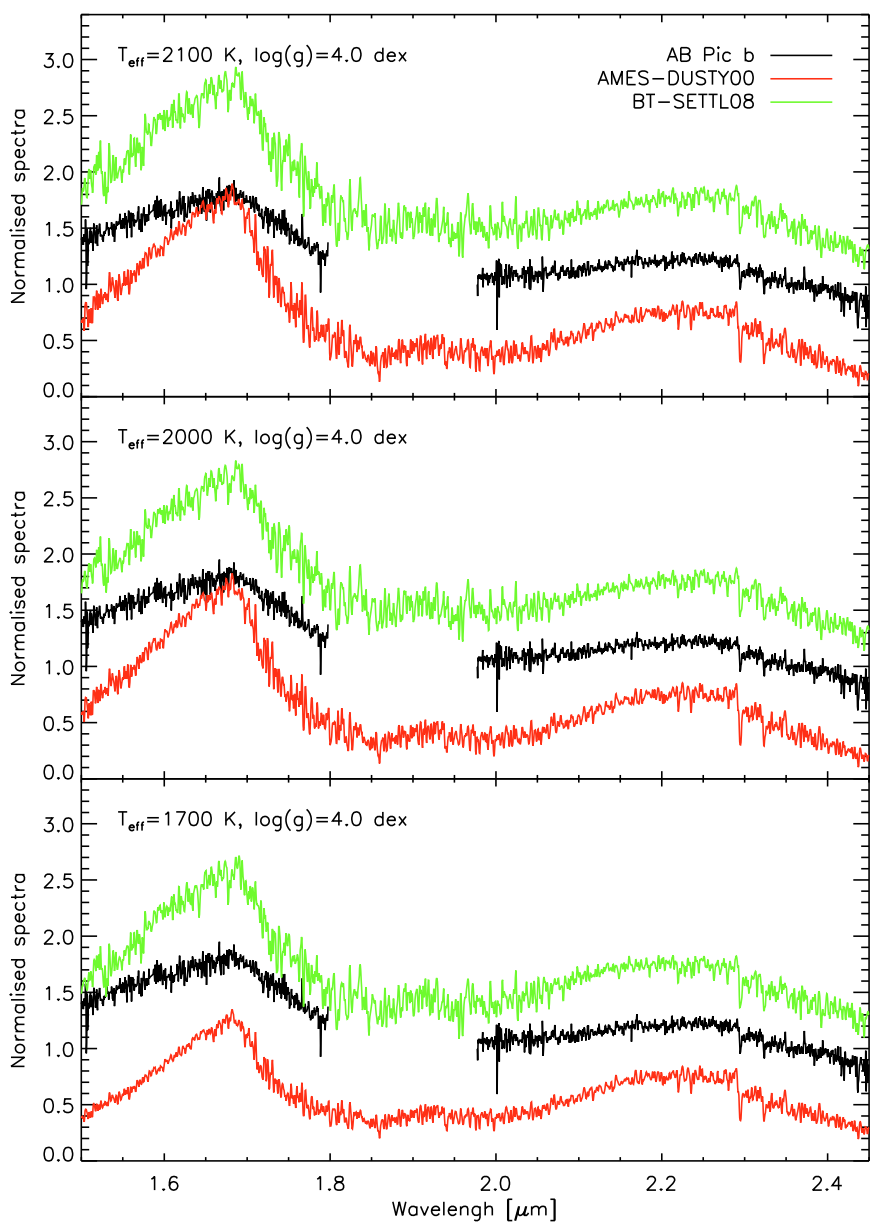

Fig. 6. Comparison of the $\mathrm{AB}$ Pic $\mathrm{b} H+K$ band spectrum to the synthetic spectra of the AMES-Dusty00 and SETTL08 libraries at $\log (g)=4.0$, $[\mathrm{M} / \mathrm{H}]=0 \mathrm{dex}$, and $T_{\text {eff }}=1700 \mathrm{~K}, 2000 \mathrm{~K}$ and $2100 \mathrm{~K}$.

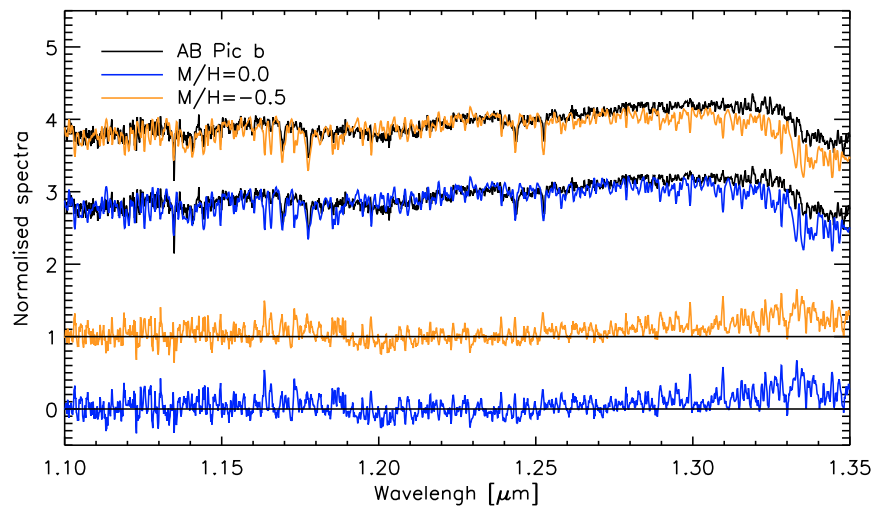

Fig. 7. Comparison of the AB Pic b $J$ band spectrum to normalized synthetic spectra (along with residuals) of the SETTL08 library at $\log (g)=4.0, T_{\text {eff }}=2000 \mathrm{~K}$, and $-0.5<[\mathrm{M} / \mathrm{H}]<0.0$.

AMES-DUSTY00 models. They find a good match for $T_{\mathrm{eff}}=$ $1700-1800 \mathrm{~K}$ and $\log (g)=4.25$. Similarily, the SETTL08 grid reproduces the observed colors for $T_{\text {eff }}=1600-1700 \mathrm{~K}$ and $\log (g)=4.0-4.5$. However, the lack of reproducibilities of synthetic spectra in the $H$ and $K$ bands revealed here shows that near-infrared colors and absolute fluxes should be used with care to infer the properties of young and cool objects. 


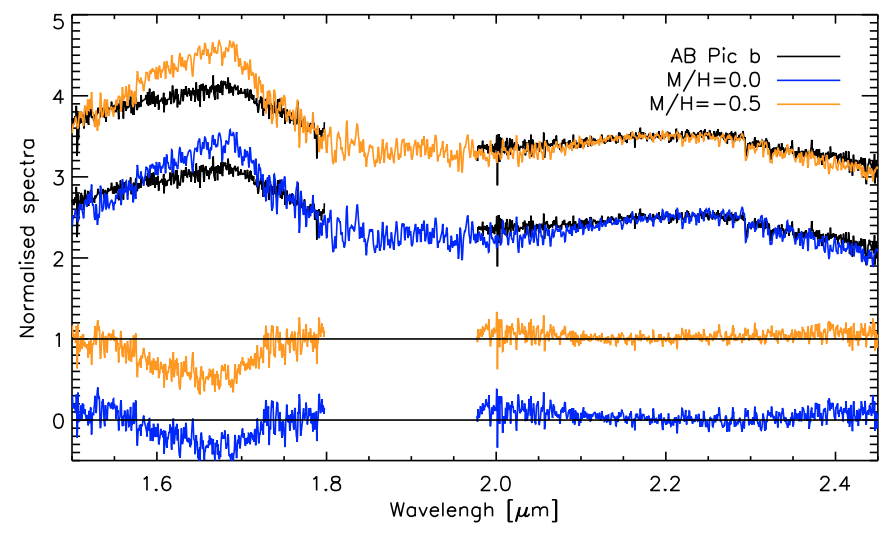

Fig. 8. Same as Fig. 7 but for the $H+K$ band.

\section{Radius, mass, and luminosity of AB Pic $b$}

\subsection{Evolutionary model predictions}

Masses of young substellar companions are mostly derived from evolutionary models predictions (D'Antona \& Mazzitelli 1997; Burrows et al. 1997; Baraffe et al. 1998; Chabrier et al. 2000; Baraffe et al. 2003; Saumon \& Marley 2008). State of the art models are based on the combination of interior models and atmospheric models necessary to link up the luminosities in observed bands to the mass, radius, effective temperatures, and surface gravities at different ages and metallicities (tracks). From the de-reddened magnitudes and colors in the 2MASS passbands, the AMES-Dusty00 evolutionary models (Chabrier et al. (2000); using AMES-Dusty00 atmospheric models) predict for AB Pic b a mass of $10 M_{\text {Jup }} \leqslant M \leqslant$ $14 M_{\text {Jup }}$ (see Table 3 ). Using $T_{\text {eff }}$ derived from our spectral analysis leads to a similar prediction of $11 M_{\text {Jup }} \leqslant M \leqslant 14 M_{\text {Jup }}$. Scarce direct mass measurements of young brown dwarfs and very low-mass stars seem to reveal that models do not achieve a good simultaneous prediction of $T_{\text {eff }}$, luminosities, radii, and masses of young low-mass objects (see Mathieu et al. 2007). Tracks need to be calibrated at young ages and very low masses down to the planetary mass regime where the formation mechanisms could actually play a key role (Marley et al. 2007). One can therefore try to use alternative methods of estimating the mass of substellar companions.

\subsection{Alternative estimations}

Based on the empirical relations between $\mathrm{BC}_{K}$ and spectral types, the luminosity of the object can be determined independently from evolutionary tracks. In the case of an L0-L1 spectral type, we derived a bolometric correction $\mathrm{BC}_{K}=3.24_{-0.19}^{+0.18} \mathrm{mag}$ from the relations of Golimowski et al. (2004) valid for field dwarfs. Luminosity, radius, and mass can then be deduced based on $T_{\text {eff }}$ and $\log (g)$ derived from our spectral analysis (see the second line in Table 3). The estimated mass agrees with evolutionary model predictions but with much larger uncertainties. However, the assumption of a similar $\mathrm{BC}_{K}-\mathrm{SpT}$ relation between young and field dwarfs has never been established and could therefore add systematic errors that are difficult to quantify.

An alternative approach has been introduced by Mohanty et al. (2004, hereafter M04) to estimate the masses, radii, and luminosities of isolated substellar objects of the Upper Sco association. The method is to use the surface flux provided by atmospheric models at the distance of the system. In the specific case of $\mathrm{AB}$ Pic $\mathrm{b}$, considering $A_{V}=0.27 \pm 0.02$
Table 3. Updated AB Pic b properties.

\begin{tabular}{cccccc}
\hline \hline Method & $\begin{array}{c}T_{\text {eff }} \\
(\mathrm{K})\end{array}$ & $\log (g)$ & $\log \left(L / L_{\odot}\right)$ & $\begin{array}{c}\text { Radius } \\
\left(R_{\text {Jup }}\right)\end{array}$ & $\begin{array}{c}\text { Mass } \\
\left(M_{\text {Jup }}\right)\end{array}$ \\
\hline Evol. & $1750_{-100}^{+100}$ & $4.2 \pm 0.2$ & $-3.6 \pm 0.2$ & $1.5-1.6$ & $10-14$ \\
$\mathrm{BC}_{K}$ & $2000_{-300}^{+100}$ & $4.0 \pm 0.5$ & $-3.7 \pm 0.2$ & $1.22_{-0.25}^{+0.70}$ & $1-45$ \\
$F_{\text {surf }-J}$ & $2000_{-300}^{+100}$ & $4.0 \pm 0.5$ & $-4.00_{-0.16}^{+0.33}$ & $0.81_{-0.2}^{+0.83}$ & $1-21$ \\
$F_{\text {surf }-K}$ & $2000_{-300}^{+100}$ & $4.0 \pm 0.5$ & $-3.72_{-0.20}^{+0.15}$ & $1.13_{-0.11}^{+0.38}$ & $2-24$ \\
\hline
\end{tabular}

(see van Belle \& von Braun 2009), the absolute magnitude in $K$-band can therefore be combined with the surface flux of the AMES-Dusty00 spectra at $T_{\text {eff }}=2000 \mathrm{~K}$ and $\log (g)=4.0$ (computed in the 2MASS filters) to determine the companion radius. From the radius and the surface gravity, we can deduce the mass, and considering in addition $T_{\text {eff }}$, we derive the luminosity. All results are reported in Table 3. The same approach can be takken using $J$-band flux, leading to slightly different values as explained by a non-simultaneous reproducibility of the $J$ and $K_{s}$-band surface flux at $T_{\text {eff }}=2000 \mathrm{~K}$. This effect has already been noticed in M04 for derivations of the radii, luminosities, and masses from $I_{C}$ and $J$ magnitudes. In both cases, estimated masses of AB Pic b again agree with evolutionary model predictions within uncertainties. The errors on these parameters results from propagated errors of the atmospheric parameters of our spectral analysis (that make the surface flux vary) and of the absolute photometry. Similar result are derived from the SETTL08 surface fluxes.

Both methods lead to consistent masses, luminosities, surface gravities, and $T_{\text {eff }}$ with evolutionary model predictions, but do not allow actually testing and refining them. Our conclusions are very sensitive to the values of $T_{\text {eff }}$ and $\log (g)$ inferred from our spectral analysis, hence adequate atmosphere spectral modeling. The strong overprediction of $\mathrm{H}_{2} \mathrm{O}$ absorptions that dominate the shape of the near-infrared spectrum of L dwarfs, even for the most recent atmospheric models, and the non-simultaneous reproducibility of the $J, H$, and $K$ bands both indicate that the comparison of the near-infrared spectra of planetary mass companions to synthetic spectra is not straightforward. It will therefore remain a difficult task for future directimaging detection and characterization, which will rely on lower resolution spectra in the near-infrared and similar libraries of synthetic spectra. For planetary mass companions at very wide orbits (no expected contamination from the primary), fitting the spectral energy distribution over a broader spectral range and directly measuring the companion luminosity with combined thermal $L$ - or $M$-band photometry or spectroscopy would certainly lead to a more robust estimation of mass and radius, as recently obtained by Leggett et al. (2008) in the case of HN Peg B.

\section{Conclusions}

We obtained high-quality $1.1-2.5 \mu \mathrm{m}$ medium-resolution spectra of the young very low-mass companion AB Pic b, whose evolutionary models place it on the planet/brown-dwarf boundary. Near-infrared spectra of young M8-L4 dwarfs were compared to the spectrum of $\mathrm{AB}$ Pic $\mathrm{b}$ using least-square fitting and spectral indexes. They confirmed the youth of the object and allowed refinement of the spectral type estimation of Chauvin et al. (2005b) to L0-L1. Our spectral classification was also confirmed by the excellent match of our spectrum to that of the young L0 field dwarf 2MASS J01415823-4633574 classified in 
the visible. A comparison to synthetic spectra enabled us to derive $T_{\text {eff }}=2000_{-300}^{+100} \mathrm{~K}$ and $\log (g)=4.0 \pm 0.5$ compatible with $0.5 \lesssim \mathrm{Fe} / \mathrm{H} \lesssim 0$. Finally, we used a bolometric correction valid for field $\mathrm{L}$ dwarfs and atmospheric models to estimate the mass, the luminosity, and the radii of the companion independently from evolutionary model predictions. The lack of bolometric corrections for young L dwarfs and the large uncertainties related to the determination of the atmospheric parameters do not currently allow the mass of AB Pic b to be refined, as is necessary for making a statement about its status and for testing evolutionary model predictions.

Our study points out the difficulties of infering young L dwarf companion properties from spectral analysis alone. Alternative methods for the evolutionary models used here rely on uncelebrated relations and atmospheric models. They need to be tested on a wide wavelength range at various gravities (age), effective temperatures, and metallicities. These loopholes will strongly limit the characterization of gaseous planets detected with the upcoming planet finders Gemini/GPI (Macintosh et al. 2006) and VLT/SPHERE (Beuzit et al. 2006, 2008). In that perspective, the XSHOOTER instrument at VLT will help acquiring simultaneous spectra over $0.3-2.5 \mu \mathrm{m}$ to robustly constrain $T_{\text {eff }}$, $\log (g)$, metallicity, and the luminosity of isolated and wide companion members of young nearby associations and star-forming regions down to the planetary mass regime. The comparison of the observed luminosity to predictions of evolutionary models will also provide a crucial constraint on evolutionary models at young ages and planetary masses, and could also clarify the role of formation mechanisms in this mass range.

Acknowledgements. We are very grateful to the anonymous referee for the constructive review that greatly improved our initial manuscript. We thank the ESO Paranal staff for performing the service-mode observations. We also acknowledge partial financial support from the Agence National de la Recherche and the Programmes Nationaux de Planétologie et de Physique Stellaire (PNP \& PNPS), in France. We are grateful to Andreas Seifahrt, David Lafrenière, and Nicolas Lodieu for providing their spectra. Finally, this work would have not been possible without the NIRSPEC (http:// wWw . astro. ucla.edu/ mclean/BDSSarchive/), IRTF (http://irtfweb. ifa.hawaii.edu/ spex/IRTF_Spectral_Library/), and SpecX (http:// www. browndwarfs.org/spexprism) libraries maintained by Ian S. McLean, Michael C. Cushing, John T. Rayner, and Adam Burgasser.

\section{References}

Abuter, R., Schreiber, J., Eisenhauer, F., et al. 2006, New Astron. Rev., 50, 398 Allard, F., Hauschildt, P. H., Alexander, D. R., Tamanai, A., \& Schweitzer, A. 2001, ApJ, 556, 357

Allers, K. N., Jaffe, D. T., Luhman, K. L., et al. 2007, ApJ, 657, 511

Baraffe, I., Chabrier, G., Allard, F., \& Hauschildt, P. H. 1998, A\&A, 337, 403

Baraffe, I., Chabrier, G., Barman, T. S., Allard, F., \& Hauschildt, P. H. 2003, A\&A, 402, 701

Barnes, J. R., Barman, T. S., Jones, H. R. A., et al. 2009, in American Institute of Physics Conference Series, ed. E. Stempels, 1094, 417

Beuzit, J.-L., Feldt, M., Dohlen, K., et al. 2006, The Messenger, 125, 29

Beuzit, J.-L., Feldt, M., Dohlen, K., et al. 2008, SPIE Conf. Ser., 7014

Bonnet, H., Abuter, R., Baker, A., et al. 2004, The Messenger, 117, 17

Bonnet, H., Ströbele, S., Biancat-Marchet, F., et al. 2003, in Adaptive Optical System Technologies II, ed. P. L. Wizinowich, D. Bonaccini, Proc. SPIE, 4839, 329

Burrows, A., Marley, M., Hubbard, W. B., et al. 1997, ApJ, 491, 856

Chabrier, G., Baraffe, I., Allard, F., \& Hauschildt, P. 2000, ApJ, 542, 464
Chauvin, G., Thomson, M., Dumas, C., et al. 2003, A\&A, 404, 157

Chauvin, G., Lagrange, A.-M., Dumas, C., et al. 2004, A\&A, 425, L29

Chauvin, G., Lagrange, A.-M., Dumas, C., et al. 2005a, A\&A, 438, L25

Chauvin, G., Lagrange, A.-M., Zuckerman, B., et al. 2005b, A\&A, 438, L29

Chauvin, G., Lagrange, A.-M., Bonavita, M.-A., et al. 2009, A\&A, 509, A52

Cushing, M. C., Rayner, J. T., Davis, S. P., \& Vacca, W. D. 2003, ApJ, 582, 1066

Cushing, M. C., Rayner, J. T., \& Vacca, W. D. 2005, ApJ, 623, 1115

D’Antona, F., \& Mazzitelli, I. 1997, Mem. Soc. Astron. Ital., 68, 807

Eisenhauer, F., Abuter, R., Bickert, K., et al. 2003, in SPIE Conf. Ser. 4841, ed. M. Iye \& A. F. M. Moorwood, 1548

Golimowski, D. A., Leggett, S. K., Marley, M. S., et al. 2004, AJ, 127, 3516

Gorlova, N. I., Meyer, M. R., Rieke, G. H., \& Liebert, J. 2003, ApJ, 593, 1074

Herczeg, G. J., Cruz, K. L., \& Hillenbrand, L. A. 2009, ApJ, 696, 1589

Kastner, J. H., Zuckerman, B., Weintraub, D. A., \& Forveille, T. 1997, Science, 277, 67

Kirkpatrick, J. D. 2005, ARA\&A, 43, 195

Kirkpatrick, J. D., Barman, T. S., Burgasser, A. J., et al. 2006, ApJ, 639, 1120

Lafrenière, D., Jayawardhana, R., \& van Kerkwijk, M. H. 2008, ApJL, 689, L153

Leggett, S. K., Allard, F., Geballe, T. R., Hauschildt, P. H., \& Schweitzer, A. 2001, ApJ, 548, 908

Leggett, S. K., Saumon, D., Albert, L., et al. 2008, ApJ, 682, 1256

Lodieu, N., Hambly, N. C., Jameson, R. F., \& Hodgkin, S. T. 2008, MNRAS, 383,1385

Lowrance, P. J., McCarthy, C., Becklin, E. E., et al. 1999, ApJ, 512, L69

Lowrance, P. J., Schneider, G., Kirkpatrick, J. D., et al. 2000, ApJ, 541, 390

Lucas, P. W., Roche, P. F., Allard, F., \& Hauschildt, P. H. 2001, MNRAS, 326, 695

Luhman, K. L., Stauffer, J. R., Muench, A. A., et al. 2003, ApJ, 593, 1093

Luhman, K. L., Peterson, D. E., \& Megeath, S. T. 2004, ApJ, 617, 565

Luhman, K. L., Adame, L., D’Alessio, P., et al. 2007, ApJ, 666, 1219

Macintosh, B., Graham, J., Palmer, D., et al. 2006, SPIE Conf. Ser. 6272

Makarov, V. V. 2007, ApJS, 169, 105

Marley, M. S., Fortney, J. J., Hubickyj, O., Bodenheimer, P., \& Lissauer, J. J. 2007, ApJ, 655, 541

Mathieu, R. D., Baraffe, I., Simon, M., Stassun, K. G., \& White, R. 2007, in Protostars and Planets V, ed. B. Reipurth, D. Jewitt, \& K. Keil, 411

McLean, I. S., McGovern, M. R., Burgasser, A. J., et al. 2003, ApJ, 596, 561

Mentuch, E., Brandeker, A., van Kerkwijk, M. H., Jayawardhana, R., \& Hauschildt, P. H. 2008, ApJ, 689, 1127

Mohanty, S., Basri, G., Jayawardhana, R., et al. 2004, ApJ, 609, 854

Mohanty, S., Jayawardhana, R., Huélamo, N., \& Mamajek, E. 2007, ApJ, 657, 1064

Nordström, B., Mayor, M., Andersen, J., et al. 2004, A\&A, 418, 989

Perryman, M. A. C., Lindegren, L., Kovalevsky, J., et al. 1997, A\&A, 323, L49

Saumon, D., \& Marley, M. S. 2008, ApJ, 689, 1327

Scholz, A., Coffey, J., Brandeker, A., \& Jayawardhana, R. 2007, ApJ, 662, 1254

Slesnick, C. L., Hillenbrand, L. A., \& Carpenter, J. M. 2004, ApJ, 610, 1045

Smith, B. A., \& Terrile, R. J. 1984, Science, 226, 1421

Song, I., Zuckerman, B., \& Bessell, M. S. 2003, ApJ, 599, 342

Stelzer, B., \& Neuhäuser, R. 2000, A\&A, 361, 581

Tinetti, G., \& Beaulieu, J.-P. 2009, in IAU Symposium, 253, 231

Tokunaga, A. T., \& Kobayashi, N. 1999, AJ, 117, 1010

Torres, C. A. O., da Silva, L., Quast, G. R., de la Reza, R., \& Jilinski, E. 2000, AJ, 120,1410

Torres, C. A. O., Quast, G. R., de La Reza, R., da Silva, L., \& Melo, C. H. F. 2001, in Young Stars Near Earth: Progress and Prospects, ed. R. Jayawardhana \& T. Greene, ASP Conf. Ser., 244, 43

Torres, C. A. O., Quast, G. R., da Silva, L., et al. 2006, A\&A, 460, 695

Torres, C. A. O., Quast, G. R., Melo, C. H. F., \& Sterzik, M. F. 2008, Young

Nearby Loose Associations, ed. B. Reipurth, 757

van Belle, G. T., \& von Braun, K. 2009, ApJ, 694, 1085

Viana Almeida, P., Santos, N. C., Melo, C., et al. 2009, A\&A, 501, 965

Vidal-Madjar, A., Désert, J.-M., Lecavelier des Etangs, A., et al. 2004, ApJL, 604, L69

Wallace, L., \& Hinkle, K. 2001, ApJ, 559, 424

Zuckerman, B., \& Song, I. 2004, ARA\&A, 42, 685

Zuckerman, B., \& Webb, R. A. 2000, ApJ, 535, 959

Zuckerman, B., Song, I., \& Webb, R. A. 2001, ApJ, 559, 388 